\title{
A Collaborative Learning Tool in Herbal Medicine for ASEAN Economic Community
}

\author{
Verayuth Lertnattee, Sinthop Chomya, and Virach Sornlertlamvanich
}

\begin{abstract}
To increase economic for countries of Southeast Asian, the ASEAN Economic Community (AEC) will be established. Herbal medicine is a part of healthcare service, which is set as important products in this community. Healthcare practitioners including pharmacists need knowledge of herbal medicine. However, it is hard for a student to familiar with several medicinal herbs with a limitation of time. In this paper, KUIHerbRx2013, a Web-based supplement learning tool on herbal medicine, is introduced. KUIHerbRx2013 supports a collaborative learning to improve knowledge and skill in herbal medicine with a scientific method and support herb names in multi-lingual and multi-script for AEC. Activities of collecting, contributing new opinions, vote to existing opinions, and providing useful information to the system, enhance skill in herbal medicine. With the assessment, the results show that this tool is the useful collaborative learning tool in herbal medicine for AEC.
\end{abstract}

Index Terms-Collaborative learning, herbal medicine, KUIHerb, ASEAN economic community.

\section{INTRODUCTION}

To increase economic for countries of Southeast Asian, the ASEAN Economic Community will be established. Herbal medicine is a part of healthcare service, which is set as important products in this community. Pharmacists including other healthcare professionals now need a basic knowledge of these topics for their professional practices [1], [2]. To serve this need, several courses on herbal medicine, e.g., general botany, medicinal botany, etc., are set in pharmacy curriculum. It is hard for a student to familiar with medicinal herbs with a limitation of time for study. Normally, students learn herbal medicine in both lecture and practice classes. In a practice class, some activities should be assigned to students such as taking pictures of some medicinal herbs or interview traditional practitioners. Students usually report the result into the simple file and print them out when they need. Using these conventional techniques, it is hard to find the way, which students to create a community for exchanging their information. With Web 2.0 system, it provides an opportunity for sharing information from a group of members

Manuscript received June 25, 2013; revised August 23, 2013. This work was supported in part by the National Science and Technology Development Agency (NSTDA) under project number P-09-00159 as well as the Research and Development Institute, Silpakorn University via research grant SURDI $53 / 01 / 12$.

Verayuth Lertnattee and Sinthop Chomya are with the Faculty of Pharmacy, Silpakorn University, Nakorn Pathom, 73000, Thailand (e-mail: verayuth@su.ac.th, verayuths@hotmail.com, sinthop@su.ac.th).

V. Sornlertlamvanich is with the National Electronics and Computer Technology Center, Pathumthani, 12120, Thailand (e-mail: virach.sornlertlamvanich@nectec.or.th). on a topic of interest [3], [4]. The Knowledge Unifying Initiator for Herbal Information (KUIHerb), a system for collective intelligence on herbal medicine, is used as a platform for building a Web community for collecting the intercultural knowledge [5]. Information in the KUIHerb has been collected for a period of time. Therefore, at least three reasons that it is not suitable to use as a learning tool. Firstly, it has only a little room for a non or a little experienced student to share a new opinion. Secondly, pharmacy students should contribute herbal information related with scientific evidences. Finally, several errors in content may occur during the learning process. The consequent is that members and visitors of the KUIHerb, may receive incorrect information. In this paper, we present an idea for building a new and clean Website based on KUIHerb and use it as a Web-based and social network learning tool in herbal medicine, which is called KUIHerbRx2013. Information of herbs in several regions can be distributed and exchanged among groups of students. Information about herb names are collected in Thai local names and multi-lingual names especially languages in ASEAN. Furthermore, opinions given to KUIHerbRx2013 should be evidence-based opinions, i.e., a set of reliable references should be cited.

In the rest of this paper, Section II gives a detail of the ASEAN Economic Community. Learning herbal medicine in school of pharmacy is described in Section III. Section IV presents KUIHerbRx2013, a learning tool in herbal medicine for AEC. The experimental setting and results from students are described in Section V and Section VI, respectively. A conclusion and future works is made in Section VII.

\section{ASEAN ECONOMIC COMMUNITY}

Southeast Asia is a sub-region of Asia, consisting of the countries that are geographically between the Indian Ocean and the Pacific Ocean. This area consists of 11 countries, i.e., Brunei Darussalam, Cambodia, Indonesia, Laos, Malaysia, Myanmar, Philippines, Singapore, Thailand, Vietnam and the newest country, East Timor. The former 10 countries have been formed into a geo-politic and economic organization which is called The Association of Southeast Asian Nations (ASEAN).

At the $12^{\text {th }}$ ASEAN Summit in January 2007, the leaders of the 10 ASEAN member countries agreed to hasten the establishment of the ASEAN Economic Community (AEC) by 2015. The ASEAN leaders adopted the ASEAN Economic Blueprint at the $13^{\text {th }}$ ASEAN Summit on 20 November 2007 in Singapore to serve as a master plan. The 10 member countries of AEC are all countries of the Southeast Asia except East Timor. The goal of establishing 
AEC is to transform the region into a single market and production base with a highly competitive advantage to make it a stable and prosperous region. For single market and production base, five core elements (free flows) are as follow: goods, services, investment, capital and skilled labors. Medical products and services is one of high-ranking priority in this community. These included traditional medicine, massage and herbal products [6]

According to information on Wikipedia [7], several languages are used in this region. Some countries have their own languages such as Thai, Laos, Khmer and etc. There are Four official languages in Singapore, i.e., English, Mandarin Chinese, Malay and Tamil. The English language is widely used for communication among countries in this region. The Chinese languages are also widely understood due to immigration of Chinese people to this region. However, several local dialects of Chinese language have been used in this region. The Southern Min language is a family of Chinese languages which is popular in this region. Three main dialects for the Southern Min language are Hokkien, Teochew and Hinanese. Traditional Chinese medicine is also familiar in ASEAN. The names of herbs may be referred by several languages. The detail of this topic will be more described in the next section.

\section{LEARNING HERBAL MEDICINE IN SCHOOL OF PHARMACY}

Herbal information is a special type of information dealing with medicinal herbs. In a pharmacy curriculum, a series of courses about herbal medicine are arranged. In these periods of study, students should be familiar with several herbs in both fresh form and dried form. Due to a limitation of time, it is hard for a student who living in a city or urban area to familiar with medicinal herbs. Normally, students learn herbal medicine in both lecture and practice classes. In a practice class, professors usually prepare some parts from medicinal plants for students. They study from these samples and some pictures, which are used for describing the dominant features of herbs. Some topics such as name identification and medicinal uses, which may be different among cultures, are still problems. Another way to make students familiar with herbs, they should see the whole plant and take pictures of those plants in both whole plants and their parts used. A set of images of an herb is excellent sources for sharing knowledge about herb identity. From the images, students can discuss, which species (including varieties) it should be. The scientific name of an herb and its images are used for common understanding. However, scientific names are not usually used as common term in communication between healthcare professionals and patients. In Thailand, Traditional Thai Medicine (TTM) has been developed for a long time. Information about herbal medicine and other types of practices is preserved and developed. Besides the TTM, Traditional Chinese Medicine (TCM) is also popular in Thailand. In a Chinese drug store, the names of crude drugs are called in Chinese languages, normally in Teochew (Chaozhou) dialect. However, the standard dialect of Chinese languages, Mandarin, is increasing in popularity among this region [8]. Information of herbs and crude drugs can be found in the Chinese pharmacopoeia including English names, pharmaceutical names [in Latin], Chinese names by Chinese characters and Romanization (Pinyin) and scientific names. It is hard for a new generation pharmacist to recognize herbs or crude drugs with these dialects. However, several Chinese herbs and other natural products are not found in Thailand. There is no Thai name for these herbs and products. The name in Chaozhou and Mandarin dialects are still important to use for identifying a Chinese herb or a crude drug.

For more understanding in herbal medicine, each pharmacy student is assigned to search and collect herbal information for some particular herbs. Several mechanisms are used to acquire and collect information, for example

- Learning from some courses in herbal medicine.

- Knowledge from their experiences.

- Interview some experienced traditional practitioners

- Seeking information from several sources, e.g., standard references, proceedings, journal including information on the Internet or library. This is the most scientific and reliable method.

At this moment, pharmacy students should be studied more about herbal medicine. Due to AEC will be coming, students may be faced with a harmonized healthcare system. For raw material about herbs and herbal products, students should be able to understand their names in several languages. Traditional including modern applications of herbal medicine for each country should be studied. Moreover, information in herbal medicine provided to professionals and patients should be based on scientific evidences.

Using a Web-based learning tool is an excellent method for sharing and exchanging herbal information in several regions and languages including dialects. The most important topic is herb identification. The way which is used for collecting herb names in several languages and dialects. The other topics are indications, methods for preparation, precautions and toxicities, additional information as well as references to opinions contributed to the community.

\section{KUIHERBRX2013: A LEARNING TOOL IN HERBAL MEDICINE FOR AEC}

At present, the first version of KUIHerb, has collected herbal information for four years. Several opinions of herbs are presented to public. For this reason, it has only a little room for a non or a little experienced student to share a new opinion. Furthermore, pharmacy students should contribute herbal information related with evidences from scientific background. However, the structure of KUIHerb is very valuable. We can establish a new and clean Website and use it as a learning tool for herbal information creation. This KUIHerb's version, which is used as a tool for a supplement learning herbal medicine, is called KUIHerbRx2013. Three types of information creation, i.e., initial, voting and non-voting types, are constructed in the Website.

\section{A. Initial Type}

The initial information is created and provided by professors. Three topics are initialized, i.e., scientific and general names (both Thai and English), general 
characteristics and references. Professors select a set of medicinal herbs and assign some of them to students.

\section{B. Sharing Opinions with Voting System}

The voting mechanism is widely used to improve accuracy of the system such as in [9]. For more accurate information, only registered students (and the administrator) of the system are able to contribute and modify their opinions. Any opinions or suggestions are committed to voting. While opinions may be different, majority votes determine the view of the communities. These features naturally realize the online collaborative works to create the knowledge communities.

A set of higher weight opinions for each topic, tends to be more believable. Three topics are applied by voting system, i.e., local names, medicinal usages and images of herbs. The voting system for local names and medicinal usages is explicit. It is represented by a voting score. A member can vote once for each opinion. On the contrary, voting system for herb image is an implicit hit counter. It is applied for summarizing the frequency of zoomed images by members. Therefore, a member may give several hits for an image. When data from these topics has been collected for a period of time, several data mining techniques, e.g., association rules [10], may be applied for finding valuable knowledge in herbal medicine.

\section{Local Names and Multi-lingual Names}

Information is usually used for identifying an herb is usually the scientific names and its pictures, which can be used for identification. However, local people know an herb with its local names. Name confusion may cause several serious problems. The thing should be kept in their mind is that an herb may have several names for each naming system and one name may be referred to several herbs. The relationship between herbs and their names is many-to-many. The KUIHerbRx can be used as an excellent tool for gathering local names and multi-lingual names. These terms can be applied in an herbal dictionary that is useful for herbal search engine.

\section{Medicinal Usages}

Medicinal uses may be different among cultures. For example, ginger is fried and eaten plain, and used in curry pastes in India. In Indonesia, it is grilled and used to flavor fish and meats or for making ginger tea. KUIHerbRx is used for pharmacy students who concern to create link between beliefs of the communities to scientific methods. From this reason, opinions are given here should cite to reliable sources of information, e.g., scientific research, standard textbooks.

\section{E. Images of Herbs}

In this system, images of an herb can be uploaded to the system. Students are assigned to take their own pictures of herbs (from real herbs). The images should relate to the whole plant and the parts which are important for identifying (e.g., leaves, flowers). The parts which have medicinal usages should also be included. This is very useful to other students who would like to see parts used of an herb. The part used should be in both fresh and dry forms. The voting system may summarize the popularity and quality of the images. The basic idea is that images, which high quality and/or useful for identifying should be more popular. To make these images more reliable, keywords and contributors' names should be given to the system: keywords suggest visitors about focus points on the image. Contributors' names guarantee visitors for quality of their images. Comments from professors and other students help owners to provide more quality images. In case of an image has some problems, e.g., incorrect picture, an image is not clear. This comment can be used as a tool to inform visitors. The owner and administrator may have a decision to keep or delete the image.

\section{F. Sharing Opinions with Non-Voting System}

Two topics are separated from the others. These are precaution/toxicity and additional information. These two topics are free text without majority voting. For a precaution, any suggestions will be kept for warning when someone would like to use the herb. For additional information, other valuable information such as cultivation may also be given.

\section{G. References for Each Opinion}

This space can be applied for suggesting references for an opinion in order to make the opinion more reliable. In KUIHerb, this topic is allowed only an administrator. However, students can access the topic in KUIHerbRx2013. When students contribute their opinions in other topics, each component should be cited by a set of reliable information sources, e.g., scientific research paper, standard text books, and etc.

\section{EXPERIMENTAL SETTINGS}

The third year pharmacy students in faculty of pharmacy, Silpakorn University that registered the course "health informatics" in the academic year of 2012 (June, 2012 to May, 2013), were assigned to share their opinions in KUIHerbRx2013. The number of students was 195. The KUIHerbRx2013 was initialed with information of 844 herbs. The 200 herbs began with information on all topics to use as samples. The rest were initialed in topics of scientific name, English names and general characteristics. Two assignments were given to students: 1) each student was an initiator on two herbs (assigned by a professor) in all topics (herb image is an optional) as much as possible and 2) the student should do the questionnaire about the KUIHerbRx2013. The students were encouraged to contribute local names in Thai and multi-lingual names especially, languages in ASEAN. For local names in Thai, the location which the name is used, has more four levels, i.e., region, province, city and distinct while only one level, i.e., country for other languages. The period of two weeks is assigned for these assignments. Students are advised to take photographs by themselves. Opinions provided to the KUIHerbRx2013 should be evidence-based information and avoid copyright violations.

\section{EXPERIMENTAL RESULTS}

\section{A. The KUIHerbRx2013}

The homepage of the KUIHerbRx2013 composes of four components, i.e., information access, information providing, Website's statistics and information sharing. For information 
access, information of an herb can be reached by two methods i.e., keyword search and directory search. The KUIHerbRx2013 provides the ability to keyword search by using a Thai common name, a local name (in Thai and other languages), an English name, a scientific name of an herb, a family name as well as an indication. It also provides the ability to browse categories of part used and symptom.

Two approaches are constructed for providing herbal information. The first approach is the current news about herbal information by Web links. The other approach, information of an herb is randomly selected from KUIHerbRx2013 database when users visit the homepage of the Website. It also provides a list of new herbs added to the database. The hit counters roughly indicate Websites' relative popularity and users' activities. Three set of counters are created for these proposes. The first set is for herbal database activity. The second set is for describing the members of the community, i.e., the number of member, the newest member, the number of active members of that time. The last statistical set reports the total activities in a period of a day, a month and a year.

In KUIHerbRx2013, seven topics are taken into account, i.e., general characteristics, pictures, local name, medicinal usages (i.e., part used with their indications and methods for preparation), precaution/toxicity, additional information (extra information) and references. Among these topics, a voting system is implemented on local names, medicinal usages and herb images. A student may choose to work individually by posting his/her opinions about those topics. Any opinions or suggestions are committed to voting. In this version, all members are given equal weight. If other students agree with the opinion, a simple click on the button "Vote" will increase the score by one. The opinion with higher score will be moved up to upper part of the window. The other three topics are separated from the others. These are precaution/toxicity, additional information and references. These three topics are free text without majority voting.

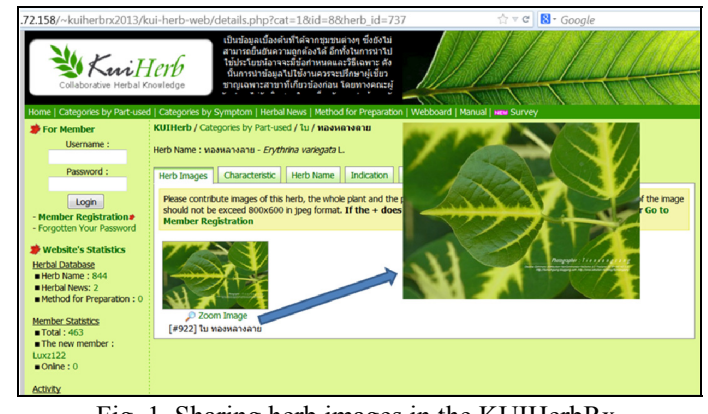

Fig. 1. Sharing herb images in the KUIHerbRx.

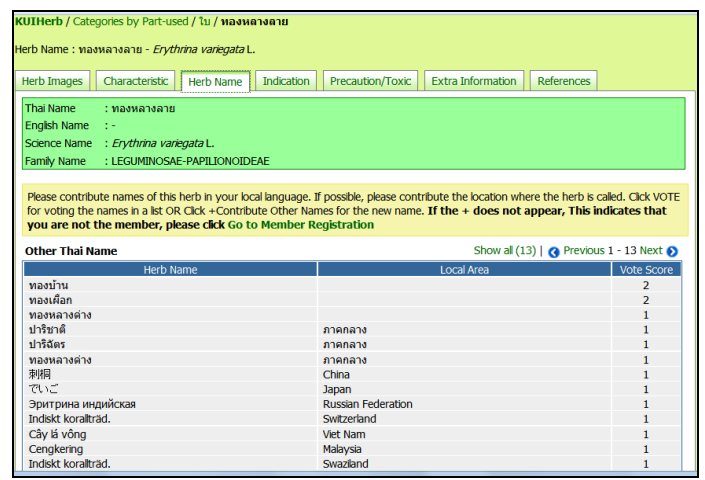

Fig. 2. Sharing multi-lingual herb names in the KUIHerbRx.
Each student was assigned to be a member of the system. Therefore, he/she could contribute and modified his/her information. The KUIHerbRx2013 was a learning system, information or opinions given to the system should be supported by reliable references. Students were assigned to contribute opinions in five topics, i.e., herb names, medicinal usages, precaution/toxicity, additional information and references. The contribution of herb images was an optional because it was hard to find some herbs in the local area. Moreover, some parts of herbs might appear in short period of time, e.g., flower and fruit. However, images of herbs have been transferred from the KUIHerb to the KUIHerbRx2013. Fig. 1 presents an image contributed by a member of the KUIHerb and this image was transferred into KUIHerbRx2013. Fig. 2 to Fig. 4 present contributed opinions of the same herb in other topics from a student. For local names and medicinal usages, a student who was assigned to be a creator for two herbs, contributed his/her opinions for these herbs. The other students could contribute their new opinions or vote for the existing opinions.

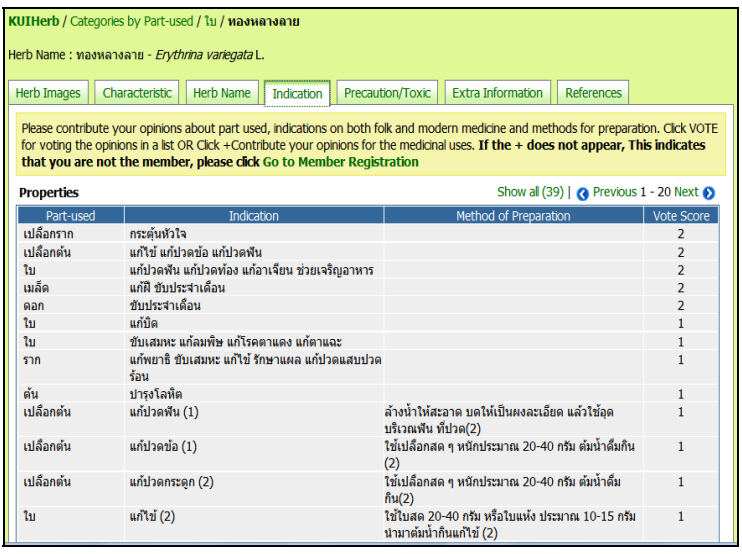

Fig. 3. Sharing medicinal usages in the KUIHerbRx

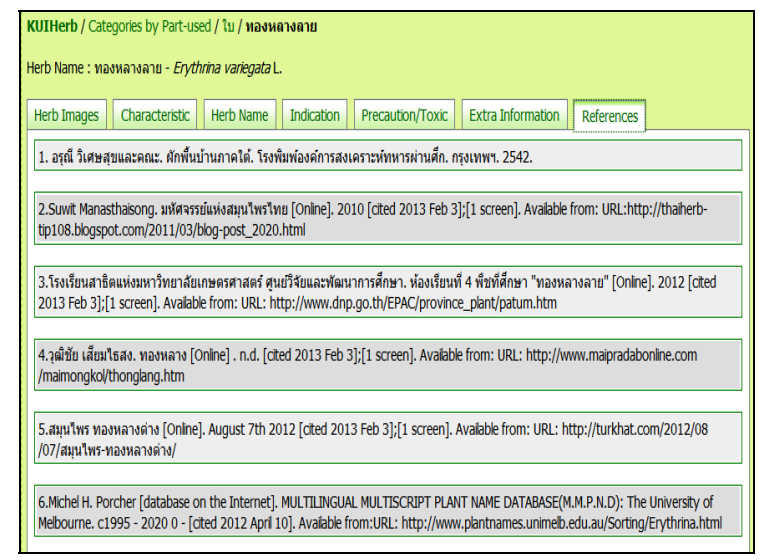

Fig. 4. Sharing references for contributed information in the KUIHerbRx

The Thai local names and herb names in several languages were contributed. This is shown in Figure 2. Some observations can be made as follow. For the languages other than Thai, several methods are used to input information about herb names. The first method is using characters of the language to present the herb name as shown in Figure 2. The second method is Romanization. The Hanyu Pinyin has been used officially to Romanize Mandarin, which is often used to write the herb name in Mandarin dialect. For example, an herb in scientific name Ocimum americanum L., the Chinese 
name is 灰罗勒, which is Romanized to "huiluole". The third method is transliteration which is a method for conversion of a text from one language to another. This method is usually used for converting Chinese characters to Chaozhou dialect in Thai. For example, an herb called ราชดัด (Thai), Brucea javanica (Linn.) Merr. (scientific name), 苦参子 (Chinese characters), is converted to โค้วเซียมจี้ (Chaozhou dialect in Thai). The last method is combination of the above methods. For example, 苦参子（โค้วเซียมจี้） is the combination of Chinese characters with transliteration. Students searched information of herb name on the Internet. A popular Website for multi-lingual herb names is "MULTILINGUAL MULTISCRIPT PLANT NAME DATABASE" from the University of Melbourne, Australia which is also appeared in the last reference in Fig. 4.

Fig. 3 presents the topic of medicinal usages of an herb. For each opinion, the references about indications and methods for preparation are given in parenthesis. For precaution/toxicity and additional information, students should contribute their opinions with references to support their opinions. A list of references is shown in the topic of references (Fig. 4).

\section{B. Contributed Information from Students}

The 195 third year pharmacy students (academic year of 2012) were assigned to contribute their knowledge in a scientific evidences. The result is shown in Table I. Two patterns of statistics are taken into account, i.e., the total opinions and the total herbs. The total opinion (Opinion) is the numbers of opinions contributed from the students for each topic. The total herb (Total Herb) is the numbers of herbs that students provide information for each topic. Note that in the topic of local and multi-lingual name, the numbers of herb names with languages other than Thai is shown in parenthesis. Furthermore, the result of herb names grouped by country, is shown in Table II.

From the results, some observations can be made. The students can collaborative work and contribute knowledge on all topics. The topic of medicinal usage was the most contributed knowledge. Several references in herbal medicine had been suggested. For local and multi-lingual names, a large number of names were given in Thai and other languages. This indicated that one herb have several names. From the Table II, several herb names in ASEAN languages were also provided. Due to the fact that pharmacy students do not have strong background in linguistics, some patterns of herb names in other languages, were provided but not a consistent pattern. In the topic of medicinal usages, a large number of herbs were given opinions. Many suggestions about precaution/toxicity and additional information were also contributed.

TABLE I: DISTRIBUTIONS OF CONTRIBUTED INFORMATION FROM STUDENTS

\begin{tabular}{lll}
\hline \hline Topic & Opinion & Total Herb \\
\hline Local and Multi-lingual Name & $1834(1225)$ & 322 \\
Medicinal Usage & 2598 & 353 \\
Precaution/Toxicity & 260 & 162 \\
Additional Information & 478 & 237 \\
References & 1292 & 381 \\
\hline \hline
\end{tabular}

TABLE II: DisTRIBUTIONS OF HERB NAMES IN ASEAN COUNTRIES

\begin{tabular}{ll}
\multicolumn{2}{c}{ INCLUDING CHINA } \\
\hline \hline Country & Opinion \\
\hline Thai & 609 \\
China & 162 \\
Malaysia & 93 \\
Vietnam & 78 \\
Indonesia & 39 \\
Philippines & 29 \\
Laos & 15 \\
Myanmar & 12 \\
\hline \hline
\end{tabular}

\section{Survey in KUIHerbRx 2013}

In the KUIHerbRx2013, a questionnaire which assessed the students' satisfaction and other opinions was used. The number of students who response to the questionnaire was 174. Three types of questions were applied. The first type, 10 questions presented by a five-point Likert item, i.e., the scale on each question is divided to 5 levels from 1 (least satisfaction or least importance) to 5 (most satisfaction or most importance). The result is shown in Table III. The second type was a set of check box, given by a respondent on the topics of sharing in the KUIHerbRx2013 which topics he/she thought that they were very useful. The last type was an open question. They can suggest anything about the KUIHerbRx2013 to make it more suitable for a learning system in herbal medicine for AEC.

TABLE III: RATINGS FOR WEBSITE DESIGN AND USE ABILITY

\begin{tabular}{|c|c|c|c|c|c|c|}
\hline \multirow[t]{2}{*}{ Topics } & \multicolumn{6}{|c|}{ Rating Score } \\
\hline & 1 & 2 & 3 & 4 & 5 & Avg. \\
\hline 1. Collective Intelligence is important & 0 & 0 & 15 & 131 & 28 & 4.07 \\
\hline 2. Level of interest in herbal medicine & 0 & 3 & 66 & 80 & 25 & 3.73 \\
\hline 3. Public community gets benefit from collaboration in KUIHerbRx & 0 & 0 & 17 & 124 & 33 & 4.09 \\
\hline $\begin{array}{l}\text { 4. Data collection in KUIHerbRx is a part of cultural preservation for herbal } \\
\text { medicine }\end{array}$ & 0 & 0 & 25 & 103 & 46 & 4.12 \\
\hline 5. Scientific evidences for each opinion are important & 0 & 0 & 17 & 118 & 39 & 4.13 \\
\hline 6. The topic about herb local names and multi-lingual names supports AEC & 0 & 2 & 32 & 109 & 31 & 3.97 \\
\hline 7. Contributions of opinions from members is an important mechanism & 0 & 0 & 14 & 110 & 50 & 4.21 \\
\hline $\begin{array}{l}\text { 8. The KUIHerbRx is able to use as a tool to support learning herbal } \\
\text { medicine }\end{array}$ & 0 & 0 & 16 & 99 & 59 & 4.25 \\
\hline 9. The KUIHerbRx supports students to familiar with herbs & 0 & 0 & 21 & 104 & 49 & 4.16 \\
\hline $\begin{array}{l}10 . \text { The ability to apply the concept of collaborative work for the future } \\
\text { work }\end{array}$ & 0 & 0 & 25 & 120 & 29 & 4.02 \\
\hline
\end{tabular}

From Table III, all questions except the question 2, gain the average scale $>3.90$. In summary, the KUIHerbRx2013 
is an important and useful tool for supporting on herbal medicine study and also preserving cultural knowledge. The numbers of respondents who special interested in herb images, local names, medicinal usages and precaution/toxicity are $79,73,148$, and 100 , respectively. Several suggestions were made. One suggestion which recommended by several respondents was that video presentations should be added into system, especially for the methods of preparation in the topic of medicinal usages. Another suggestion asked for the user interface of the Website should have more languages (it has two languages, i.e., Thai and English).

\section{CONCLUSION AND FUTURE WORKS}

In this work, the KUIHerbRx2013 was used as a Web-based supplement learning tool in herbal medicine for AEC. Herbal medicine is a part of healthcare service, which is set as important products in this community. Due to the diversities of herbs, geographic distribution and their applications, social network was important for enhanced learning. KUIHerbRx2013 provides a supplement learning to improve knowledge and skill in herbal medicine with a scientific method and support multi-lingual and multi-script herb names. Information of medicinal herbs in several regions can be distributed and exchanged among groups of students. Several topics were assigned to students, e.g., local and multi-lingual names, medicinal usages, precaution/toxicity, additional information and references. Activities of collecting, contributing new opinions or vote to exist opinions, and providing comments to the system, enhanced skill in herbal medicine. Students gained more experience on herbal medicine. Skill in searching information using traditional method such as books or modern technology such as the Internet, was improved.

In the KUIHerbRx2013, some problems to provided multi-lingual multi-script herb names were found. The structure of tables in a database and the front end for local and multi-lingual name should be redesigned and implement in both the KUIHerb and KUIHerbRx. These will be implemented for our future work.

\section{REFERENCES}

[1] L. Dvorkin, P. Gardiner, and J. S. Whelan, "Herbal medicine course within pharmacy curriculum," Journal of Herbal Pharmacother, vol. 4, no. 2, pp. 47-58, January 2004.

[2] G. Murtaza et al., "An evaluation of Pakistani pharmacy students knowledge of herbal medicines in Pakistan," Afr J Pharm Pharmacol, vol. 6, no. 3, pp. 221-224, January 2012.

[3] K. J. Lin, "Building web 2.0," Computer, vol. 40, no. 5, pp. 101-102, May 2007
[4] T. Gruber, "Collective knowledge systems: Where the social web meets the semantic web," J Web Semant: Science, Services and Agents on the World Wide Web, vol. 6, no. 1, pp. 4-13, February 2007.

[5] V. Lertnattee, S. Chomya, and V. Sornlertlamvanich, "Applying collective intelligence for search improvement on Thai herbal information," in Proc. $9^{\text {th }}$ International Conference on Computer and Information Technology, Xiamen, 2009, pp. 178-183.

[6] S. Supakankunti and C. Herberholz, 'Transforming the ASEAN Economic Community (AEC) into A Global Services Hub: Enhancing the Competitiveness of the Health Services Sectors in Thailand' in Tullao, T. S. and H. H. Lim (eds.), Developing ASEAN Economic Community (AEC) into A Global Services Hub, pp.147-171, 2012.

[7] Southeast Asia. (2013). [Online]. Available: http://en.wikipedia.org/wiki/Southeast_Asia.

[8] R. J. L. Polla, "Language contact and language change in the history of the sinitic languages," Procedia - Social and Behavioral Sciences, vol. 2, no. 5, pp. 6858-6868, 2010.

[9] Y. Ko, J. Park, and J. Seo, "Using the feature projection technique based on a normalized voting method for text classification," Inform Process Manag, vol. 40, pp. 191-208, 2004.

[10] X. Luo, K. Yan, and X. Chen, "Automatic discovery of semantic relations based on association rule," $J$ Soft, vol. 3, no. 8, pp. 11-18, November 2008.

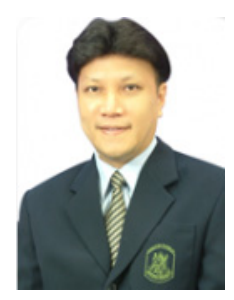

Verayuth Lertnattee was born in Bangkok, Thailand 1965. He received a bachelor degree in pharmacy, master degree in science (computer science) from Chulalongkorn University in 1989, 1996, respectively. $\mathrm{He}$ also received a master degree in Science (Pharmacy) from Mahidol University in 1991. He received a Ph.D. in Technology from Sirindhorn International Institute of Technology, Thammasat University, His research interests include data mining in medical, herbal, pharmaceutical information, information retrieval and collective intelligent. He is currently an associate professor in department of health-related informatics, faculty of pharmacy, Silpakorn University, Thailand.

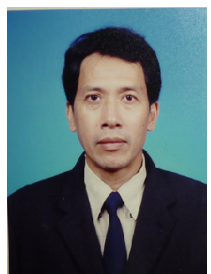

Sinthop Chomya was born in Suphanburi, Thailand 1955. He received a bachelor degree in pharmacy, master degree in Pharmacy (Pharmaceutical Botany) from Chulalongkorn University in 1980, 1984, respectively. His research interests include phytochemistry and Thai traditional medicine. He is currently an associate professor in department of pharmacognosy, faculty of pharmacy, Silpakorn University, Thailand.

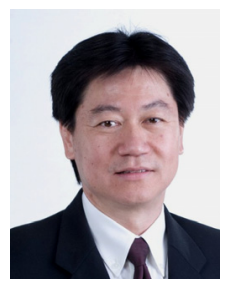

Virach Sornlertlamvanich was born in Thailand 1960. He received his bachelor and master degrees in engineering from Kyoto University in 1984 and 1986 respectively, and doctoral degree in Computer Science from Tokyo Institute of Technology in 1998. His current research interests include natural language processing and information technology. He is assigned as a director of Asia-Pacific Association for Machine Translation (AAMT), an Executive committee member of Asian Federation of Natural Language Processing (AFNLP), and a technical committee member of many international academic conferences and journals such as IJCNLP, COLING, MT Summit, ACL, LREC, PACLIC, GWC, IEICE, TALIP, etc. He is currently Principal Researcher of National Electronics and Computer Technology Center (NECTEC), Thailand. 